and Diehl review what is known about the neurobiology of Attention Deficit Hyperactivity Disorder and commonly used measures for diagnosis. Stimulant medications, non-stimulant medication and psychosocial interventions are all discussed as treatment strategies. In a discussion of executive dysfunction, Mahone and Slomine have included a table of 16 evidence-based investigations with information that includes intervention(s) used and results. Another table of nonpharmacological intervention strategies for initiation, planning and organization, inhibitory control, and working memory is divided into age groups so that appropriate recommendations may be offered for preschoolers, elementary school students, and adolescents. In addition to the above, a chapter by Klein-Tasman, Phillips, and Kelderman on genetic syndromes associated with intellectual disability provides numerous references for treatment strategies given a variety of syndromes such as Fragile-X, Down, Angelman, and Prader-Willi. The second section concludes with several chapters devoted to the pathophysiology, classification, and management of pediatric movement disorders.

The third section explores broad-based interventions that, for the most part, have not been previously discussed. In a chapter on quantitative electroencephalography and neurofeedback, Krull, George, and Strother provide the reader with basic information about each approach as well as with results on investigations performed thus far. Given a conclusion that both interventions lack appropriate evidence to support their use, they have provided an outline of the criteria necessary for an approach to meet adequate empirical standards. In a chapter devoted to neuroimaging, Bigler uses traumatic brain injury as an exemplar to posit that structural and functional imaging not only aids in diagno- sis, but also helps monitor recovery and/or one's response to intervention. His cases are well illustrated with scans that have color counterparts in the Plate section at the end of the text. Owley uses autistic-spectrum disorders as a model to discuss the challenges of using medication in the treatment of a neurodevelopmental disorder and its target symptoms. The author's point that the field of pediatric psychopharmacology is in its infancy makes it ripe for study. The final two chapters of the third section discuss cognitive rehabilitation of pediatric brain injury. According to Butler, this is a field left largely to educators given shrinking insurance coverage. A cognitive remediation program is presented; however, like psychopharmacology, this is an understudied area. As such, there is a paucity of empirically based intervention strategies for cognitive rehabilitation.

While Pediatric Neuropsychological Intervention covers a broad range of disorders, there are some omissions. For example, myelomeningocele is not covered; and pediatric stroke is given only scant mention. Regardless, the text has much to offer. For example, opening with a discussion on basic development, and the need for parents and educators to be involved in the process of assessment reinforces the notion that the best practice of pediatric neuropsychology is an upward rather than downward extension. Additionally, the text provides background information on many of the disorders seen in both inpatient and outpatient clinical practice. Finally, it contains the most up-to-date research in the area of intervention for children and adolescents. The finding that some populations have not been studied as extensively as others ideally will drive future research, a goal of the Editors. Overall, there is no doubt that this resource will more often be off rather than on the bookshelf.

\title{
Neurotherapeutics: Alternative Career or at Least Novel Information
} doi: $10.1017 / \mathrm{S} 135561770808065 \mathrm{X}$

\section{Progress in Neurotherapeutics and Neuropsychopharmacology. Volume 1. Jeffrey L. Cummings (Ed). 2006. Cambridge, England: Cambridge University Press, 169 pp., \$122.00 (HB).}

Reviewed by Jim Andrikopoulos, Ph.D., ABPP, Ruan Neurology Clinic, Des Moines, Iowa, USA.

Progress in Neurotherapeutics and Neuropsychopharmacology provides an overview of what is for most neuropsychologists a new content area-clinical trials. Had I not spent five years in the pharmaceutical industry, I probably would have not given the topic of neurotherapeutics a second thought. This book can be useful on a number of counts. First, knowledge of neurotherapeutics is an indirect method for understanding the basic science of neurological diseases since drug development hinges upon appreciating the pathology of the disease. Second, three factors make neuropsychologists uniquely qualified to understand neu- rotherapeutics in the form of clinical trials-knowledge of most neurological disease states and research training. Finally, all clinical trials have a primary outcome measure. When the primary outcome measure is cognition, our contribution as neuropsychologists can be paramount. My introduction to neurotherapeutics was as outcome rater. An increasing number of neuropsychologists are being recruited by the pharmaceutical industry to work in medical affairs, mostly as medical science liaisons. For those interested in a career alternative, or the topic of neurotherapeutics, this text provides a starting point. 
The Introductory chapter by Jeffrey Cummings provides a nice, succinct overview of the process by which drugs are brought to market. The remaining chapters describe clinical trials for various neurologic disease states, including psychiatric conditions. Chapter 1 , by Tekin and Lane, is a straightforward chapter about rivastigmine, the first acetylcholinesterase inhibitor to be approved by the FDA for Parkinson's dementia. Chapter 2, by Stankoff et al., addresses treatment of fatigue in multiple sclerosis (MS). A cursory literature review yielded nearly 600 publications in which MS appeared in the title and fatigue in the abstract. The trial that they describe is the largest doubleblind-placebo-controlled trial with the drug modafinil. Despite this large literature, there is a lack of knowledge about the pathophysiology of fatigue in MS, which may be why this trial was negative. Of particular interest was the large placebo effect, suggesting non-organic factors may explain the fatigue. The placebo effect is an endemic problem in many clinical trials.

Two chapters illustrate an important issue concerning drug efficacy. The chapter by Mason et al. addresses a large multicenter phase III trial that randomized patients diagnosed with glioblastoma to radiotherapy alone or radiotherapy with temozolomide. Based on this trial the FDA approved the drug as first line treatment for glioblastoma. Unfortunately, the primary outcome of overall survival only increased by $2 \frac{1}{2}$ months. The chapter by Gordon et al. describes two small early phase trials of minocycline in amyotrophic lateral sclerosis (ALS). These are safety and tolerability trials that predate the pivotal phase III trials that result in drug approval. The only ALS medication currently approved by the FDA is riluzole. Like temozolomide for glioblastoma, riluzole prolonged life by two months. These results are illustrative of a practical point about drug efficacy. Despite statistical significance, the clinical and real-world significance of clinical trial results is questioned. ALS is also featured in two additional chapters. Shefner provides an overview of a negative small trial of creatine in ALS, and Pope looks at AVP-923, a novel agent in the treatment of pseuodobulbar affect; the clinical trial was successful and the medication appears to be on the verge of approval under the brand name, Zenvia.

It is not uncommon for medications approved for one condition to be pursued for an FDA indication for another disease in a completely different therapeutic area. As example, two anti-epileptic agents are also approved for migraine prophylaxis (i.e., topiramate $\&$ divalproex sodium). In the chapter by Hollander et al., fluoxetine, an antidepressant, is used to successfully treat repetitive behaviors associated with childhood autism. Two chapters that break the mold are those by Wiendeles \& Ferrari on migraine, and by Meltzer on schizophrenia. The migraine chapter is not likely to be of interest to neuropsychologist but it does illustrate methodological considerations involved in clinical trial design. The schizophrenia chapter introduces the novel concept of a "metatrial", a cost-effective way of bringing drugs to market. In this trial design, multiple novel agents are tested in a small trial. If one is successful then larger trials are pursued with the successful agent.

The last three chapters are likely to be of less interest to practicing neuropsychologists. One of these, a chapter by Simpson et al., looks at the efficacy and tolerability of ziprasidone and olanzapine in patients with schizophrenia and schizoaffective disorder. Head-to-head studies (i.e., comparing one active agent against another) are generally not done by pharmaceutical companies, as one runs the risk of funding a study that may show that the competitor's drug is superior. Commonly, head-to-head studies are undertaken when drugs that trail in the marketplace challenge the market leader. A good example of this was when an interferon beta 1a MS agent, Rebif, challenged Avonex, another interferon beta 1a agent, voiding Avonex's exclusivity in the U.S. marketapparently the first time the FDA allowed this.

There are three caveats to this book. First, in the Introduction, by Cummings, a disclosure statement is appropriately included that lists the pharmaceutical companies for which this author has served as a consultant so as to disclose any conflicts of interest. Such disclosure statements are now compulsory in medical journals and prior to presentations at medical meetings. The remaining chapters do not contain similar disclosures. Employees of pharmaceutical companies authored a couple of the chapters. As an example of possible bias, the authors of these two chapters do not discuss any limitations of their trials, in contrast to some of the non-industry affiliated authors. Journal articles require disclosure of the funding source for the study. Only one chapter lists the funding source as a pharmaceutical company. Hopefully this will be corrected in future volumes. Second, this book needs to offer something more than a description of the trial or trials since it would be cheaper to obtain the trial article versus buying the book. Lastly, one wonders why these trials were chosen among other trials. There are trials that are more notable and controversial. There is a conspicuous absence of stroke trials, which are conducted at twice the rate of the next two most common therapeutic areas (i.e., Parkinson and Alzheimer's disease) combined. Although neuroprotection trials in stroke have been a dismal failure, the heuristic value of neuroprotection, across nearly all therapeutic areas, is evidenced by researchers determined to have a positive trial. There have been over 100 failed neuroprotection trials in neurology. Another absorbing topic can be found in functional neuroimaging and Parkinson's disease. There has been a contentious debate, largely driven by the pharmaceutical industry, pitting the dopamine agonists against levodopa, suggesting that the agonists may be neuroprotective (i.e., CALM-PD study) and levodopa toxic.

The exposure to neurotherapeutics provided by this book may result in a neuropsychologist more readily appreciating that a pivotal clinical trial in the New England Journal of Medicine can impact their understanding of that neurological disease, sometimes more than an assessment article in our journals. For me, this has been the case for a number of years. 